\title{
Using Statistical and Artificial Neural Networks to predict the Permeability of Loosely-Packed Granular Materials
}

\author{
Faiz M. Mahdi ${ }^{(1,2, *)}$ and Richard G. Holdich ${ }^{(1)}$ \\ ${ }^{(1)}$ Department of Chemical Engineering, Loughborough University, Loughborough \\ LE11 3TU, UK, \\ ${ }^{(2)}$ Chemical Engineering Department, Sirte University, Sirte, Libya \\ *Correspoding author's email: faizmaa@hotmail.co.uk
}

\begin{abstract}
Well-known analytical equations for predicting permeability are generally reported to overestimate this important property of porous media. In this work, more robust models developed from statistical (MVR) and Artificial Neural Network (ANN) methods utilized additional particle characteristics ('fines ratio' $\left(\mathrm{x}_{50} / \mathrm{x}_{10}\right)$ and particle shape) that are not found in traditional analytical equations. Using data from experiments and literature, model performance analyses with average absolute error (AAE) showed error of $\sim 40 \%$ for the analytical models (Kozeny-Carman and HappelBrenner). This error reduces to $9 \%$ with ANN model. This work establishes superiority of the new models, using experiments and mathematical techniques.
\end{abstract}

Keywords: Loosely-Packed Granular Materials, Multivariate Regression, Artificial Neural Network and Permeability Prediction 


\section{Introduction}

The permeability of solids forming a porous medium is an important parameter that determines the frictional loss during fluid flow through that medium. It can be used to predict the flow rate of fluid through the material for a given pressure difference when modelling the behaviour of fluid transport in any porous media, or packings. In many filtration experiments in the laboratory, the objective is to determine the filter cake permeability or, alternatively, specific cake resistance to filtration and how it varies with pressure as well as information on the filter medium resistance after it has stabilised $[1,2]$. The permeability of the porous media is essential to establish the relationship between the fluid flow rate and pressure gradient for application of Darcy's law [3].

In practice, permeability is a function of various parameters including particle size, particle shape, voidage and packing arrangement, particle size distribution and on occasion the concentration of slurry being filtered [4]. Theoretical relations for permeability are often used as a guide to estimate permeability if no operating data is available. Measured permeabilities may be one or even two orders of magnitude lower than that given by the analytical models [5]. In general, the permeability of porous media is measured experimentally using Darcy's law, which is the basic equation relating pressure drop $(\Delta P)$ and superficial velocity $\left(U_{0}\right)$. For a single dimension this is described by Eq. (1):

$$
\frac{\Delta P}{L}=-\frac{\mu}{k} U_{0}
$$

where $k$ is the filter cake permeability $\left[\mathrm{m}^{2}\right], \mu$ is the liquid viscosity $[\mathrm{Pa} s]$ and $L$ is the bed height [m]. Darcy's law is often applied to a single-phase fluid flow in a porous 
medium when the flow is laminar [6]. The permeability of a liquid such as water, through a porous sample can be measured simply by allowing it to flow continuously through the sample with a constant pressure gradient [6]. The volume of liquid that flows through the sample is measured at specific time intervals, which can be used to calculate the volumetric flow rate $[7,8]$.

The porous medium refers to a body of particles usually irregular in shape, different in size, vary in size distribution and have different surface morphology. These characteristics affect the industrial processes in different ways and their effects make particle characterisation a difficult but important application in many manufacturing processes. In industry, particle shape and size are the most important factors, which affect particulate system behaviour such as flow and reaction properties $[9,10]$. In particular, particle characteristics such as particle size, particle size distribution (especially the fine particles), solid concentration, particle shape and orientation influence the permeability of a porous medium $[11,12]$.

There are a number of well-known analytical expressions for the prediction of permeability e.g. Happel-Brenner (H-B) Eq. (2) and Kozeny-Carman (K-C) Eq. (3) but their reliability is limited [13-15].

$$
\begin{aligned}
& k=\frac{\left(2-3 C^{1 / 3}+3 C^{5 / 3}-2 C^{2}\right)}{3+2 C^{3 / 2}} \frac{x_{s v}^{2}}{12 C} \\
& k=\frac{(1-C)^{3}}{K} \frac{x_{s v}^{2}}{36 C^{2}}
\end{aligned}
$$

where $k$ is the hydraulic permeability $\left[\mathrm{m}^{2}\right], C$ is the solid concentration by volume $(C=1-e)$ where $e$ is the voidage and $x_{S V}$ is the Sauter mean diameter [m], $K$ is the Kozeny coefficient which may be equal to 5 as is conventionally assumed $[16,17]$ in 
the absence of any experimental work to determine an empirical alternative value, which would be the case when performing design type calculations.

The $\mathrm{K}-\mathrm{C}$ and $\mathrm{H}-\mathrm{B}$ models assume that the particles are in fixed geometry, rigid and are in point contact with each other [4]. It has been reported that both models work best within a voidage range of $0.4<\varepsilon<0.7$ [18]. Also, the K-C model and many other models contain 'coefficients' whose values often depend on the process and material properties and as such there exist no relationships for their predictions [17].

The determination of permeability through experiments is time consuming and is not always possible. The available models for its prediction are limited and to overcome these challenges, there is a need to develop new models that allow the prediction of permeability using different variables under various process conditions. This is the motivation for this work; it aims to establish a new model, or technique, that can predict permeability at very low and high solid concentrations, and also for different sizes and shapes of particles.

\section{Computational Methods}

\subsection{Multivariate Regression (MVR)}

The MVR model is one of the most widely used of all statistical methods. MVR accommodates more than one response variables and that can be useful when variability in the independent variable occurs. Also, MVR models such as PCR and PLSR, are based on the inverse method [19-21]. The PLSR and PCR approaches are both linear and similar with the only main difference, which is the way that the data is compressed. In PCR the regression is applied to those variables that account 
for variance in the input data, but in PLSR variance is present in both (input and output) and this is considered during model building. As a result, the compressed variables obtained will be different and are termed as latent variables $[19,22]$.

In a number of studies comparing MVR and ANN models the values of root mean square error (RMSE) have been used to determine which technique fits best. The ANN models showed better fit as they are capable of catching sophisticated nonlinear integrating effects $[21,23,24]$. In one comprehensive study, Saleemi, (2011) [25], the PCR model failed to predict the output accurately and a significant difference was observed between the measured and predicted values. The MVR model faces difficulties when multicollinearity exists in the data. Multicollinearity occurs when some of the variables can be expressed as linear functions based on other variables in the system. This limits the MVR capability and leads to an unstable model with poor predicted response [25]. Johnson and Wichern, (2007) [26] reported that the published applications of multivariate methods have increased tremendously in recent years to include data reduction or simplification, sorting and grouping, investigation of the dependence among variables, prediction and hypotheses testing.

\subsection{Artificial Neural Networks (ANN)}

ANN within MATLAB is an interconnected assembly of simple processing elements (units or nodes) whose functionality is based on the structure and function of biological neural networks with ability to learn from rounds of training, using existing data. Thus, it is a very useful modelling tool with neurons operating in parallel, typically in three layers; input, hidden and output [27-31]. An ANN can be trained to 
perform a particular function by adjusting the values of the weights assigned to the neurons between the inputs and the output [32-34].

In the literature, ANN model is still being designed in a time-consuming iterative trial and error method, which depends mostly on the problem itself and the user's experience [35]. Also, ANN can be designed based on either single or multilayer network [36]. The network system can either be Feedforward (FF) or Feedback (FB) $[27,29,36]$. ANN with the FB algorithm is the most popular and is very common in engineering applications [37-39]. Although, many transfer functions can be used with ANN, the most commonly used are log-sigmoid, tan-sigmoid and linear [29, 40, 41].

ANN modelling begins with network training. There are different algorithms for training, and it is difficult to identify the fastest and most accurate one [42]. Supervised and unsupervised training methods are used [40, 43]. However, a supervised training algorithm has more applications [34, 37]. To improve the model performance, a high number of data points needs to be used for the training set [43]. Training stage is followed by validation and testing of the network to evaluate performance [29].

\subsubsection{Advantages and Disadvantages of ANNs:}

The main advantages of using ANNs are: it requires basic level programming and it is becoming widely accepted to simplify programming and algorithm design for a given wide range of outputs [28, 36, 44]. ANNs are particularly useful for solving problems that cannot be expressed as a series of steps, such as series prediction 
and data mining $[31,45]$. The utilization of an ANN approach has been reported previously in the prediction of permeability for the purposes of petroleum reservoir engineering [46], but this was not based on particle characterisation such as size distribution and shape. On the other hands, according to Agachi et al., (2006) [47], ANNs have limited ability to identify possible causal relationships, as there is no established criteria for interpreting the weights and biases. When dealing with a large number of variables ANNs are likely to over fit $[31,45]$.

Zargari et al., (2013) [31] states that, permeability is one of the most difficult physical properties to predict because it is based on number of parameters that are not easy to determine either experimentally or theoretically. For example, the size of particle, which has the highest influence on the prediction of permeability, is measured based on spherical shaped calculation while in reality it can be away from that e.g. clay. Permeability can be time and cost consuming to obtain from laboratory data and is prone to subjective interpretation. Instead of using traditional regression techniques, ANNs provide accuracy, consistency and improved overall quality of permeability prediction for reservoir engineering [48]. Wakeman and Wu, (2003) [49] established a relationship for the specific cake resistance and the combined cake resistance using an ANN model for vibration assisted filtration. This model consists of three inputs (vibration acceleration, cumulative filtrate and concentration), using one hidden layer (8 neurons) and one output (vibration specific cake). They found that ANNs showed promising results, but this method also has its own disadvantages such as failing to succeed when limited analysis data are available [37]. In general, using a large number of data points increases the processing time and decreases the impact of missed data [46]. More recently, Pazuki et al., (2012) [42] studied the 
efficiency and accuracy of the ANN model for prediction of oil reservoir structure as a key parameter in reservoir engineering. Various Multilayer Perceptron (MLP) models, with different learning algorithms, layers and node numbers, were investigated. Their results show that, ANN with Improved Back Propagation (IBP) learning method and five nodes in the middle layer gave the highest accuracy for their applications.

In order to make a rational decision about methods of computational intelligence, Zargari et al. (2013) [31] compared predicted permeabilities, again, for the purposes of reservoir engineering, from ANN and Adaptive Neuro-Fuzzy Inference System (ANFIS). Results showed that, the ANN model was much more accurate than the ANFIS. In addition, Tahmasebi and Hezarkhani (2012) [46] investigated the performance and accuracy of two different permeability prediction methods: Modular Neural Network (MNN) and traditional Multilayer Perceptron (MLP). The obtained permeability results showed that incorporating MLP showed good prediction. In the case of soil compaction and groundwater engineering Sinha and Wang (2007) [50] concluded that ANN prediction models could be used for compression and permeability determination with sufficient accuracy for their purposes, but again this was not work based on fundamental particle characterisation. It is apparent that ANNs have been accepted widely as tools for research in petroleum reservoir engineering and Geotechnical applications, but not yet for the prediction of loosely packed granular material.

In the above work, researchers used different input parameters e.g. electrical conductivity and resistivity, photoelectric effect, solid density, bulk density, sonic transient time, spectral Gamma ray, deep induction log, correlated log porosity, 
depth and water saturation. These parameters describe the local properties of the reservoir rather than the solid itself. Also, none of these parameters have considered either the particle shape or size distribution. Their analysis was appropriate for the modelling of large consolidated oil reservoirs, but not for the particulate constituents forming the reservoir.

\subsection{Model Performance}

The efficiency of network design mostly depends on the learning algorithm, topology and data distribution, which change from one dataset to another [46]. The criteria used to evaluate the performance of a model usually are the Coefficient of Correlation $\left(\mathrm{R}^{2}\right)$, Root Mean Square Error (RMSE) and Average Absolute Error (AAE) as shown in Eq. (4), (5) and (6) respectively [42, 50].

$$
\begin{aligned}
& R^{2}=1-\frac{\sum\left(Y_{\text {measured }}-Y_{\text {pred }}\right)^{2}}{\sum\left(Y_{\text {measured }}-\frac{\sum Y_{\text {measured }}}{N}\right)^{2}} \\
& R M S E=\sqrt{\frac{1}{N} \sum_{i=1}^{i=N}\left(Y_{\text {measured }}-Y_{\text {pred }}\right)^{2}} \\
& A A E=\sum \frac{\left|Y_{\text {measured }}-Y_{\text {pred }}\right|}{Y_{\text {measured }}}
\end{aligned}
$$

where, $Y_{\text {pred }}$ is the network prediction value, $Y_{\text {measured }}$ is the experimental response value, $N$ is the total number of reading in the data points. RMSE is the basic tool to check the model accuracy $[29,32,41]$. These three measures of performance were applied for both numerical methods (MVR and ANN) while AAE was used to evaluate the analytical models ( $\mathrm{K}-\mathrm{C}$ and $\mathrm{H}-\mathrm{B})$. 
The purpose of this research is to develop an alternative approach for the estimation of porous media permeability (and therefore specific resistance or any other derivative of permeability) for various materials under different conditions from particle characterisation data and process parameters and relevant to nonconsolidated systems such as during filtration and solid-liquid separation. This alternative approach can be employed for design and modelling purposes and is based on statistical computational (MVR) techniques and Artificial Neural Network (ANN) modelling.

In this study, various approaches and techniques were used. The data were obtained from diverse sources (experimental and literature). Pre-processing of the data, investigation of the design and evaluation of numerical models were performed in order to achieve the ANN and MVR models with excellent performance. Finally, the predicted results from analytical (existing models) and numerical models (developed in this work) were examined in the light of the measured results with the aim of establishing a more reliable model.

\section{Materials and Methods}

In this study, the permeability of the porous medium constituted by particulate solids is investigated during different separation processes including: sedimentation, permeation and filtration (constant pressure and rate). For the modelling and regression based techniques the data (total of 547 points) was obtained from previous studies $[5,15,51-56]$. The results are compared with the predicted values from the particle size analysis data using analytical models ( $\mathrm{K}-\mathrm{C}$ and $\mathrm{H}-\mathrm{B})$. For the analysis of the filtration tests, the general filtration equation [16] was used to 
calculate specific cake resistance $(\alpha)$ for both constant pressure and constant rate filtration.

Furthermore, these previous studies were conducted to investigate the permeability of the porous media for different materials characterisation and process parameters using the same experimental procedure. The effect of various variables such as particle size, shape and size distribution, applied pressure and flow rate, $\mathrm{pH}$ and solid concentration on permeability were examined. All of these previous studies, which are used here, employed the same techniques as in the followed description. The Sauter mean diameter and size distributions of the particles were calculated using Malvern 2000 and Horiba LA-920 equipment laser diffraction based devices. Morphology of all materials was studied in order to find the shape coefficient of the particles, which was calculated using Heywood's approach [57]. The Multivolume Pycnometer 1305 was used to measure the density of the materials. The data input was limited to the above literature studies, which mentioned at the start of section 3, due to uncertainties when attempting to obtain data from similar studies reported in the literature; for example it is not common for authors to provide sufficient information on the shape of the particles used to enable its use in the training data set used in this work.

In MVR, there are a number of different models that can be used, but principal component regression (PCR), partial least squares regression (PLSR) and simple nonlinear regression (NLR) are common and were chosen. All of these methods are predictive model building techniques [25]. These models were found from a regression package provided by XLSTAT, which is an Add-In application for 


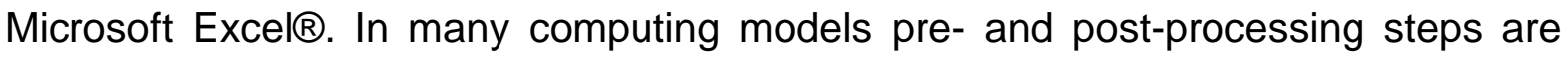
required, which lead to improved model training efficiency. There are several routines that can be used; the most common are provided automatically within the model. Both, the input and the output vectors are normalized before applying the regression algorithm [39]. From the literature, it was found that the PCA function is highly recommended for use on the data before regression analysis.

\subsection{Pre-Processing Data}

The main objectives of pre-processing data when applying ANN technique are: to remove outliers (noise) and to obtain a training data set that serves as the characteristic of the input and output data. Some outliers are the result of incorrect measurements and can be immediately rejected and removed from the set of data $[58,59]$. In this work, the raw data (547 points) was checked and cleaned statistically by removing all data (17 points) that does not follow similar data pattern. In order to find the outliers of all data, this step was down after combining all data from all sources.

\subsection{Selection of input variables}

According to Naes, (2002) [60] introducing too many factors will result in a large network size and consequently increased processing time and decreased efficiency. Understanding the influence of input variables is of primary concern when developing a numerical model [31]. In order to overcome this difficulty Principal Component Analysis (PCA) function was applied to the data and then the treated data was used as an input for the ANNs and NLR models, although, PCA is a built-in function for PCR and PLSR models. In this work, eight different variables (Sauter 
mean diameter, particle concentration, 'fine ratio' $\left(x_{50} / x_{10}\right)$, shape coefficient, shape factor (sphericity), solid density, $\mathrm{pH}$ and pressure gradient) were obtained and investigated numerically (using PCA) and experimentally [5, 51]. Table 1 shows that, particle size is the most significant variable, having a correlation coefficient of 0.92 . These results corroborate the theoretical models (e.g. K-C and $\mathrm{H}-\mathrm{B}$ ) on the importance of particle size on the porous medium permeability. In the same table, the next important parameter is particle concentration though with much lower coefficient of correlation. Two other variables worthy of consideration are the shape and the size distribution of particles.

The four variables mentioned above were identified as the effective variables in the prediction of permeability to be considered as the inputs for the numerical models. Two of these variables (particle size and concentration) are already explicitly presented in the theoretical models. The output is the measured permeability $(k)$ that is obtained from experimental methods as presented in Table 2.

\subsection{ANN Design}

In order to create a successful model, a number of investigation on different aspects is required e.g. the model design, the number of layers and the elements per layer, the connections between the layers, the transfer and training functions [61]. There are standard steps required for designing an ANN model to solve a problem as shown in Figure 1.

Different variables (e.g. inputs number, transfer, learning and training functions) affect the ANN performance, but number of hidden layers and neurons are the most 
important. The number of hidden layers is based on the number of inputs as reported in the literature. On the other hand, trial and error is used to find the number of neurons in each hidden layer. The number of neurons in the output layer corresponds to the number of output variables that are desired [39, 62]. Arpat et al., (1998) [37] stated that for reservoir permeability prediction, supervised algorithms are generally preferred. Using FB network, which is a development technique, will ease several problems. Therefore, an ANN model was designed using the FB network to determine the optimum number of layers and neurons.

To be in a good agreement with the analytical models such as $\mathrm{K}-\mathrm{C}$ and $\mathrm{H}-\mathrm{B}$, different numerical models were studied. Some of them (ANN2, NLR2) consist only of two variables (as in the analytical models) and the other (ANN4, NLR4) use four variables (from the variable investigation) as shown later in Tables 3 and 4 for ANN and in Figure 5 for NLR.

\section{Results and Discussion}

\subsection{Data Analysis}

The variables used in this study for permeability prediction covered a large range of data as shown in Table 2. Due to this extensive data collection, the data was treated using the PCA function for easy and quick conversion.

The main statistical descriptions of the data used are illustrated in Figure 2. About $90 \%$ of the particle size values are less than $10 \mu \mathrm{m}$ and almost $75 \%$ of the solid concentrations are between 0.3 and $0.5(\mathrm{v} / \mathrm{v})$. Additionally, more than $90 \%$ of the particle size distribution is less than 5 and more than $70 \%$ of the shape coefficient 
values are higher than 0.5 . The preponderance in term of data at the fine particle size is deliberate and useful. In the case of predicting permeability for design purposes, it is the prediction of finer particles permeability that poses the design problem, not that of larger particles $[4,16]$.

\subsection{ANN design and training results}

In all cases the ANN was implemented using the ANN module contained within the MATLAB environment. Initially, two different numbers of hidden layers (1 and 2) were studied to find the optimum number for the relationship between input and output data; it was found that, an ANN model with one hidden layer suffices for this purpose. Also, different numbers of neurons in the hidden layer were investigated with ANN2 and ANN4 as shown in Tables 3 and 4.

The ANN models with various transfer, training and learning functions were run. The results showed that the combination of TANSIG (tangent sigmoid), TRAINBR (bayesian-regularization) and LEARNGDM (gradient descent weight/momentum) functions yielded optimum results. The ANNs architecture of ANN4 model (with four inputs) includes one hidden layer and one output layer as shown in Figure 3. Subsequently, in the case of using only two inputs, particle size (which had the highest correlation value) was studied with shape coefficient and size distribution as well as with particle concentration in order to investigate the best two inputs for use with the models. As can be seen in Tables 3 and 4, twenty networks modelled for both ANN cases (ANN2 and ANN4). Accuracy was measured using two accepted parameters: the coefficient of determination $\left(R^{2}\right)$ and the root mean square error 
(RMSE). The text in bold indicates the optimum model. These results are shown later in Figure 6.

From all the information above, it can be seen that the designed ANN models give an acceptable performance based on both, $\mathrm{R}^{2}$ and RMSE. Furthermore, the ANN2 results in Table 3 are in good agreement with the analytical models when using only two variables (particle size and concentration) as inputs. The design of the ANN in this work is based on creating a Feedback (FB) network with one hidden layer architecture with either 4 nodes (ANN2) or 5 nodes (ANN4), in the hidden layer (see Figure 3). Of all the architectures simulated, the ANN model with only one hidden layer and five nodes showed better results than the other models.

\subsection{Predicted and measured permeability comparison}

\subsubsection{PCR, PLSR and NLR Models:}

MVR based linear models can give a stable solution when using a larger number of principal components for slightly nonlinear data, while a nonlinear model can give much better solutions using fewer variables [60]. The prediction permeability function

of the NLR model using the four input variables (NLR4) is shown in Eq. (7). This equation was found from a nonlinear regression package provided by XLSTAT, which is an Add-In application for Microsoft Excel ${ }^{\circledR}$.

$$
\begin{aligned}
& k^{*}=3.922+0.406 X_{1}-0.062 X_{2}-0.419 X_{3}+15.099 X_{4} \\
& -0.912 X_{1}^{2}+0.091 X_{2}^{2}+0.496 X_{3}^{2}+10.355 X_{4}^{2} \\
& +0.357 X_{1}^{3}-0.558 X_{2}^{3}+0.513 X_{3}^{3}-14.997 X_{4}^{3} \\
& +0.308 X_{1}^{4}+0.356 X_{2}^{4}-0.238 X_{3}^{4}-13.561 X_{4}^{4}
\end{aligned}
$$


where $k^{*}$ is the predicted output, $X$ are the responses: $X_{1}$ is the Sauter mean diameter $\left[\mathrm{m}^{2}\right], X_{2}$ is particle concentration $(\mathrm{v} / \mathrm{v}), X_{3}$ is shape coefficient, $X_{4}$ is particle spread $\left(x_{50} / x_{10}\right)$. Both Eq. (8) and (9) are used to normalize $\left(Y^{*}\right)$ and denormalize $\left(k^{\prime}\right)$ the data respectively.

$$
\begin{aligned}
& Y^{*}=\frac{2\left(X_{i}-X_{\text {max }}\right)}{\left(X_{\text {max }}-X_{\text {min }}\right)}-1 \\
& k^{\prime}=\left(\frac{\left(k^{*}+1\right)\left(k_{\text {max }}^{*}-k_{\text {min }}^{*}\right)}{2}\right)+k
\end{aligned}
$$

where $X_{i}$ is the variable value, $X_{\max }$ and $X_{\min }$ are the maximum and minimum values of the inputs respectively that are shown in Table 2 for all inputs $\left(X_{1}, X_{2}, X_{3}\right.$ and $\left.X_{4}\right), k^{*}$ is the predicted output, $k_{\max }^{*}$ and $k_{\min }^{*}$ are the maximum and minimum values of the predicted output as in Table 2 respectively.

The Eq. (7) is used to predict the value of output $\left(k^{*}\right)$ that is de-normalized using Eq. (9) before taking the anti-log10 and then multiplied with 1E-16, as demonstrated in the example of permeability prediction using NLR model shown in the Appendix. Figure 4 shows a flowchart describing this process. The RMSE and $R^{2}$ were used to quantify the prediction performances for PCR, PLSR and simple Nonlinear Regression (NLR) models. The values obtained for all of these models (linear and nonlinear) using the 4 inputs (size, concentration, shape and size distribution) are shown in Table 5. As can be seen in Figure 5 the performance criteria values for both PCR and PLSR models are similar, which could imply that these models give similar regression coefficients and prediction results as is often found in the 
literature. However, the NLR model shows much better prediction than both linear models as shown in Table 5 and Figure 5.

\subsubsection{ANN Model:}

Figure 6 presents the predicted porous media permeability from both ANN models and shows that ANN4 predicts permeability better than ANN2. Also, results of ANN4 and the analytical models were compared with the measured values (see Figure 7). This is carried out with the aim of understanding the relationship between them and to find the degree of difference. Measured results are represented by the dashed red line. Figure 7 demonstrates that the ANN model results are closer to the measured results, with reduced error when compared to the analytical models. The average absolute error (AAE, Eq. 6) was found with $\mathrm{K}-\mathrm{C}$ and $\mathrm{H}-\mathrm{B}$ models to be $35 \%$ and $40 \%$, respectively. The results of using ANN2 model provide an error ratio of $14 \%$. However, the ANN4 model decreases the error ration to approximately $9 \%$ compared to the measured results. One important reason for the reduced error is the addition of a shape coefficient and particle spread (fines ratio) in the ANN4 model. These two parameters are not inherent in the analytical relations, such as $\mathrm{K}-\mathrm{C}$ and H-B models of permeability.

A comparison of both nonlinear (NLR and ANN) models regarding RMSE and $\mathrm{R}^{2}$ values, under the same conditions (4 inputs), shows that the ANN model within MATLAB provides a better prediction, as presented in Figures 5 and 6 . However, the NLR model gives more advantages as the use of a special code is not required unlike in the case of MATLAB. In addition, the Excel software is easier to access and use compared to the MATLAB software. Furthermore, the AAE was calculated for all 
models. The results showed that, the AAE decreased significantly from about $40 \%$ to $9 \%$ by using the ANN4 with four inputs. Table 6 presents a summary of all the performance criteria for the models with their values. This table shows that, adding two more inputs improved the prediction of the nonlinear models

In general, it can be observed that the ANN model in all cases successfully maps the training data and provides more accurate prediction values of permeability. The ANN model results give a reasonable view of the link between permeability using different methods and different conditions of materials and thus provide good predictions that are better than the analytical and the MVR models. 


\section{Conclusion}

Using multivariate regressions (MVR) and artificial neural network (ANN), novel statistical models for the prediction of permeability of loosely-packed materials are presented. Existing analytical models (like K-C and $\mathrm{H}-\mathrm{B}$ ) were shown to overestimate permeability prediction leading to error in designs of process equipment. The data used were taken from both experimental tests as well as what are reliably reported in the literature where permeability of filter cakes for different materials was reported.

As a result of PCA and the experimental investigation of the input variables, this study is based on four input variables (three are inherent ones from the particle characterisation process): Sauter mean diameter (ranging from 0.2 to $168 \mu \mathrm{m}$ ), the 'fines ratio' $\left(x_{50} / x_{10}\right)$, particle shape coefficient (as based on Heywood's approach) and voidage of the porous media (ranging from 98.5 to $37.2 \%$ ). Using these four parameters as inputs, performance of models from the linear and nonlinear MVR as well as ANN were investigated together with the existing analytical models ( $\mathrm{K}-\mathrm{C}$ and $\mathrm{H}-\mathrm{B})$. The $\mathrm{K}-\mathrm{C}$ and $\mathrm{H}-\mathrm{B}$ are two-variable models (particle size and voidage) and their comparison with corresponding two-variable models from ANN (ANN2) and MVR (NLR2) showed that the latter models have better predictive performance. Furthermore, four-variable (particle size, 'fines ratio', particle shape, and voidage) models developed from the MVR (NLR4) and ANN (ANN4) exhibit excellent performance.

Based on the values of $R^{2}$, RMSE and $A A E$, the performance of different linear regression models was analysed and compared to nonlinear regression and ANN models. From this comparison, the ANN4 model showed better prediction than the 
other models. ANN4 results were compared with the results of the analytical models ( $\mathrm{K}-\mathrm{C}$ and $\mathrm{H}-\mathrm{B})$. The $\mathrm{AAE}$ was found with $\mathrm{K}-\mathrm{C}$ and $\mathrm{H}-\mathrm{B}$ models to be 35 and $40 \%$, respectively while the results of using ANN2 model reduced the AAE to $14 \%$. The ANN4 model further reduced the AAE to approximately $9 \%$ compared to the measured results. Furthermore, it was found that using the ANN4 model led to increase in the $R^{2}$ value from 0.90 to 0.99 and significant decrease in the RMSE value from 0.121 to 0.054 . The new models possess the capability to predict the permeability of porous media more accurately owing to the incorporation of the additional particle characteristics that are not found in the existing models.

It can be concluded that the ANN model with four inputs and one hidden layer with five nodes provide the most reliable prediction with better fits than the other models. This work demonstrates that ANNs are capable of catching sophisticated non-linear integrating effects. However, prediction of permeability using this ANN approach depends on the availability of the ANN code to the user, it is based on MATLAB which is an industry standard, but it is not universally available and accessible. A simpler alternative approach is to use the NLR model within Excel, using the constitutive equations provided here and the procedure illustrated in Figure 4 (and the Appendix). This provides an accessible method for the prediction of permeability in non-consolidated porous media based on the characterisation data from the particles making up that media. 
Acknowledgements: Faiz Mahdi is grateful to the Libyan Ministry of Higher Education and Sirte University in Libya, for sponsoring this work. 


\section{References}

1. Tarabara V V, Hovinga RM, Wiesner MR (2002) Constant transmembrane pressure vs. constant permeate flux: effect of particle size on crossflow membrane filtration. Environmental Engineering Science 19:343-355. doi: 10.1089/109287502320963355

2. Tiller FM (1953) The Role of Porosity in Filtration - Numerical Methods for Constant Rate and Constant Pressure Filtration Based on Kozeny Law. Chemical Engineering Progress 49:467-479.

3. Mauran S, Rigaud L (2001) Application of the Carman-Kozeny Correlation to a High Porosity and Anisotropic Consolidated Medium: The Compressed Expanded Natural Graphite. Transport in porous media 43:355-376.

4. Rushton A, Ward AS, Holdich RG (2000) Solid-Liquid Filtration and Separation Technology. 2nd:587.

5. Mahdi FM, Holdich RG (2013) Laboratory cake filtration testing using constant rate. CHERD 91:1145-1154.

6. Loosveldt $\mathrm{H}$, Lafhaj Z, Skoczylas F (2002) Experimental study of gas and liquid permeability of a mortar. Cement and Concrete Research 32:1357-1363. doi: http://dx.doi.org/10.1016/S0008-8846(02)00793-7

7. Baraka-Lokmane S (2002) Hydraulic versus pneumatic measurements of fractured sandstone permeability. Journal of Petroleum Science and Engineering 36:183-192. doi: http://dx.doi.org/10.1016/S0920-4105(02)00317-0

8. Despois J-F, Mortensen A (2005) Permeability of open-pore microcellular materials. Acta Materialia 53:1381-1388. doi: http://dx.doi.org/10.1016/j.actamat.2004.11.031

9. Rhodes M (2008) Introduction to Particle Technology. doi: 10.1002/9780470727102

10. Wijngaarden RI, Westerterp KR, Kronberg A, Bos ANR (1998) Industrial Catalysis: Optimizing Catalysts and Processes. Wiley-VCH, Germany

11. Biggs S (2006) Aggregates Structures and Solid-Liquid Separation Processes. KONA Powder and Particle 24:41-53.

12. Fu X, Huck D, Makein L, et al (2012) Effect of particle shape and size on flow properties of lactose powders. Advances in Characterization and Modeling of Particulate Processes 10:203-208. doi: http://dx.doi.org/10.1016/j.partic.2011.11.003

13. Brinkman HC (1949) A calculation of the viscous force exerted by a flowing fluid on a dense swarm of particles. Applied Scientific Research 1:27-34. doi: 10.1007/BF02120313

14. Carman PC (1956) Fluid flow through granular beds. Transactions of the Institution of Chemical Engineers 15:150-166.

15. Mahdi FM (2008) Permeability Modelling, Loughborough University MSc Thesis. Loughborough University Thesis 
16. Holdich RG (2002) Fundamentals of Particle Technology. Midland Information Technology \& Publishing, U.K

17. Tien C, Ramarao B V (2013) Can filter cake porosity be estimated based on the Kozeny-Carman equation? Powder Technology 237:233-240. doi: http://dx.doi.org/10.1016/j.powtec.2012.09.031

18. Davies $L(1980)$ Theoretical and experimental values for the parameter $k$ of the Kozeny-Carman equation, as applied to sedimenting suspensions. Journal of Physics D: Applied Physics 13:2013.

19. Bakeev KA (2010) Process Analytical Technology: Spectroscopic Tools and Implementation Strategies for the Chemical and Pharmaceutical Industries. Wiley

20. Fox J, Weisberg S (2011) Multivariate Linear Models in R, An Appendix to An R Companion to Applied Regression. Sage, Thousand Oaks, CA

21. Gosasang V, Chandraprakaikul W, Kiattisin S (2011) A Comparison of Traditional and Neural Networks Forecasting Techniques for Container Throughput at Bangkok Port. The Asian Journal of Shipping and Logistics 27:463-482. doi: http://dx.doi.org/10.1016/S2092-5212(11)80022-2

22. Gemperline P (2010) Practical Guide To Chemometrics, Second Edition. Taylor I\& Francis

23. Moradzadehfard M, Motlagh PA, Fathi MR (2011) Comparing Neural Network and Multiple Regression Models to Estimate Dividend Payout Ratio. Middle-East Journal of Scientific Research, IDOSI Publications 10:302-309.

24. Pao H-T (2008) A comparison of neural network and multiple regression analysis in modeling capital structure. Expert Systems with Applications 35:720-727. doi: http://dx.doi.org/10.1016/j.eswa.2007.07.018

25. Saleemi AN (2011) Strategic feedback control of pharmaceutical crystallization systems, Loughborough University PhD Thesis. Loughborough University

26. Johnson RA, Wichern DW (2007) Applied Multivariate Statistical Analysis. Pearson Prentice Hall

27. Brownlee J (2011) Clever Algorithms: Nature-Inspired Programming Recipes. 1st:436.

28. Da Silva IN, Flauzino RA (2008) An approach based on neural networks for estimation and generalization of crossflow filtration processes. Applied Soft Computing 8:590-598. doi: 10.1016/j.asoc.2007.03.008

29. Haykin SS (2009) Neural Networks and Learning Machines. 3rd:934.

30. Iwata M, Jami MS, Shiojiri S (2007) Artificial Neural Networks model to predicte compression-permeability characteristics of solid/liquid systems. Filtration Solutions 7:337-344.

31. Zargari H, Poordad S, Kharrat R (2013) Porosity and Permeability Prediction Based on Computational Intelligences as Artificial Neural Networks (ANNs) and Adaptive 
Neuro-Fuzzy Inference Systems (ANFIS) in Southern Carbonate Reservoir of Iran. Petroleum Science and Technology 31:1066-1077. doi:

$10.1080 / 10916466.2010 .536805$

32. Shetty GR, Chellam S (2003) Predicting membrane fouling during municipal drinking water nanofiltration using artificial neural networks. Journal of Membrane Science 217:69-86. doi: 10.1016/S0376-7388(03)00075-9

33. Wang P (2006) Artificial General Intelligence and Classical Neural Network. IEEE International Conference of Granular Computing 130-135.

34. Zabihi R, Schaffie M, Nezamabadi-pour H, Ranjbar M (2011) Artificial neural network for permeability damage prediction due to sulfate scaling. Journal of Petroleum Science and Engineering 78:575-581. doi:

http://dx.doi.org/10.1016/j.petrol.2011.08.007

35. Boozarjomehry RB, Svrcek WY (2001) Automatic design of neural network structures. Computers \& Chemical Engineering 25:1075-1088. doi:

http://dx.doi.org/10.1016/S0098-1354(01)00680-9

36. Christos S, Dimitrios S (2010) Neural Networks. Imperial College,, UK

37. Arpat GB, Gümrah F, Yeten B (1998) The neighborhood approach to prediction of permeability from wireline logs and limited core plug analysis data using backpropagation artificial neural networks. Journal of Petroleum Science and Engineering 20:1-8. doi: http://dx.doi.org/10.1016/S0920-4105(98)00034-5

38. Nowroozi S, Ranjbar M, Hashemipour H, Schaffie M (2009) Development of a neural fuzzy system for advanced prediction of dew point pressure in gas condensate reservoirs. Fuel Processing Technology 90:452-457. doi: http://dx.doi.org/10.1016/j.fuproc.2008.11.009

39. Saeedi A, Camarda K V, Liang TJ (2007) Using Neural Networks for Candidate Selection and Well-Performance Prediction in Water-Shutoff Treatments Using Polymer Gels-A Field-Case Study. SPE Prod \& Oper 22:417-424.

40. Boroumand A, Baziar M (2005) DETERMINATION OF COMPACTED CLAY PERMEABILITY BY ARTIFICIAL NEURAL NETWORKS. iwtc.info 515-526.

41. Khataee AR, Kasiri MB (2010) Artificial neural networks modeling of contaminated water treatment processes by homogeneous and heterogeneous nanocatalysis. Journal of Molecular Catalysis A: Chemical 331:86-100. doi: 10.1016/j.molcata.2010.07.016

42. Pazuki GR, Nikookar M, Dehnavi M, Al-Anazi B (2012) The Prediction of Permeability Using an Artificial Neural Network System. Petroleum Science and Technology 30:2108-2113. doi: 10.1080/10916466.2010.512888

43. Jeff TH (2005) Introduction to Neural Networks with Java. 1st:380.

44. Graupe D (1999) Principles of Artificial Neural Networks. 3:252.

45. Jeff TH (2008) Introduction to Neural Networks for C\#. Heaton Research, Inc. 
46. Tahmasebi P, Hezarkhani A (2012) A fast and independent architecture of artificial neural network for permeability prediction. Journal of Petroleum Science and Engineering 86-87:118-126. doi: http://dx.doi.org/10.1016/j.petrol.2012.03.019

47. Agachi PS, Nagy ZK, Cristea MV, Imre-Lucaci A (2006) Model Based Control: Case Studies in Process Engineering. doi: 10.1002/9783527609475

48. Wiener J, Rogers J, Moll B (1995) Predict permeability from wireline logs using neural networks. Petroleum Engineer International 68:18-24.

49. Wakeman RJ, Wu P (2003) Neural Network Modelling of Vibration Filtration. Filtration $3: 237-244$

50. Sinha SK, Wang MC (2007) Artificial Neural Network Prediction Models for Soil Compaction and Permeability. Geotechnical and Geological Engineering 26:47-64. doi: 10.1007/s10706-007-9146-3

51. DiGiovanni BA, Mahdi FM, Starov VM, Holdich RG (2012) Particulate clusters and permeability in porous media. CHERD 90:1168-1176.

52. Holdich RG, Tarleton ES, Shaw FJ (1993) A new experimental technique for the analysis of cake filtration. Filtech Exhibitions/ The Filtration Society 11-20.

53. Tarleton ES, Hancock DL (1997) Using mechatronics for the interpretation and modelling of the pressure filter cycle. Chemical Engineering Research and Design 75:298-308.

54. Tarleton ES, Willmer SA (1997) The effects of scale and process parameters in cake filtration. Chemical Engineering Research and Design 75:497-507.

55. Tarleton ES, Wakeman RJ (1999) Software Application in filter control, data acquisition and data analysis. Filtration \& Separation 36:57-64.

56. Tarleton ES (1999) Using mechatronics technology to assess pressure filtration. Powder Technology 104:121-129.

57. Heywood H (1971) Particle size and shape distribution for lunar fines sample 12057,72. Proceedings of the Lunar Science Conference 3:1989-2001. doi: 1971LPSC....2.1989H

58. Devore JL (2011) Probability and Statistics for Engineering and the Sciences. Cengage Learning, Boston, USA

59. Walpole RE, Myers RH, Myers SL, Ye K (2012) Probability and Statistics for Engineering and the Sciences. Pearson Education, USA

60. Naes T (2002) An User-friendly Guide to Multivariate Calibration and Classification. Nir Publications

61. Anderson D, McNeill G (1992) Artificial Neural Networks Technology. 1-83. 
62. Zuluaga E, Alvarez HD, Velasquez JD (2002) Prediction of Permeability Reduction by External Particle Invasion Using Artificial Neural Networks and Fuzzy Models. Journal of Canadian Petroleum Technology 41: 


\section{Nomenclature}

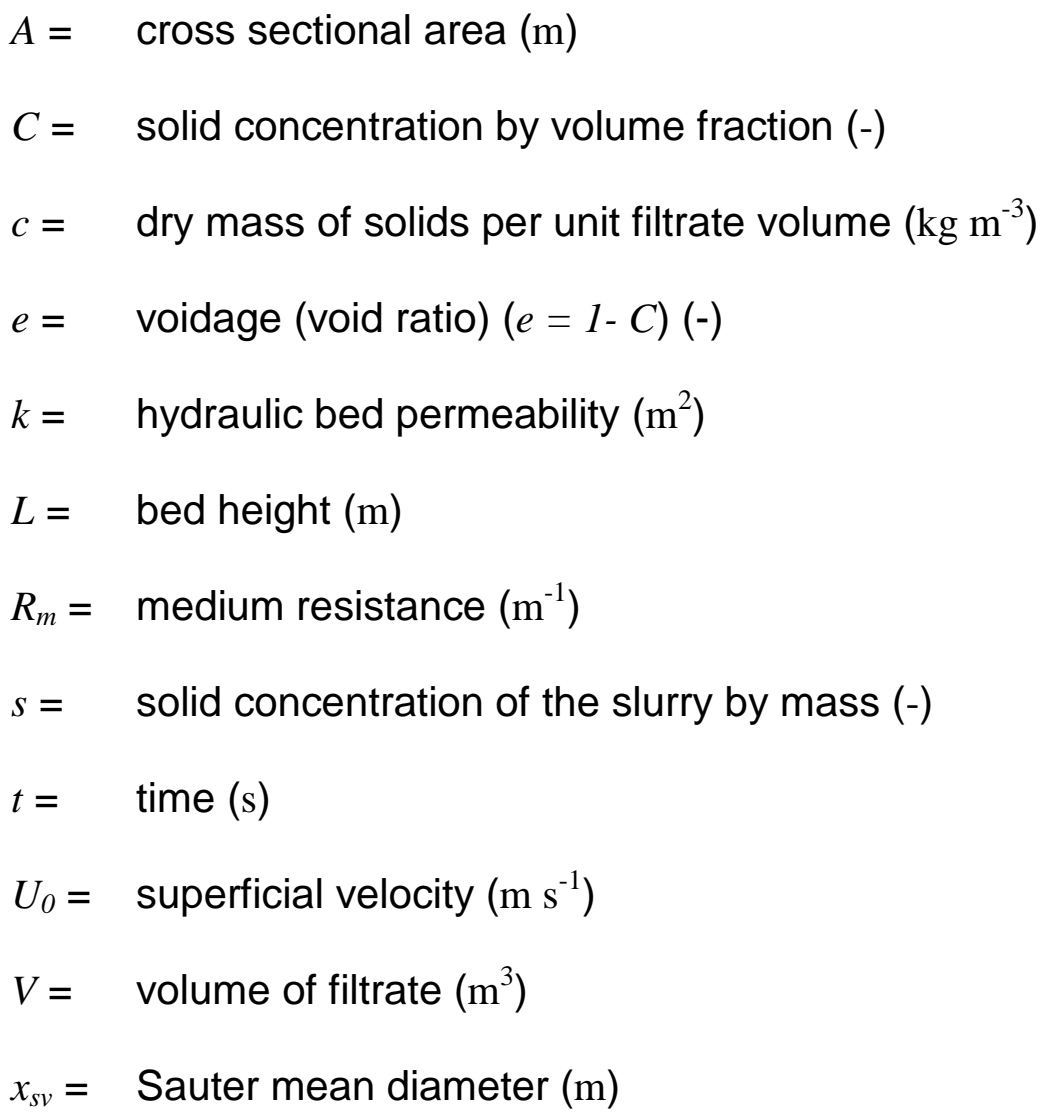




\section{Abbreviations}

$\mathrm{AAE}=$ Average Absolute Error

ANN $=$ Artificial Neural Networks

ANN2 $=$ Artificial Neural Networks consisting of two inputs

ANN4 = Artificial Neural Networks consisting of four inputs

ANFIS = Neuro-Fuzzy Inference System

NLR2 $=$ Simple Nonlinear Regression model consisting of two inputs

NLR4 = Simple Nonlinear Regression model consisting of four inputs

$\mathrm{FF}=$ Feedforward network

FB $=$ Feedback network

$\mathrm{IBP}=$ Improved Back Propagation

H-B = Happel-Brenner Model

$\mathrm{K}-\mathrm{C}=$ Kozeny-Carman Model

RMSE $=$ Root Mean Squared Error

$\mathrm{R}^{2}=$ Coefficient of Correlation

MVR = Multivariable Regression

MNN = Modular Neural Network

MLP = Multilayer Perceptron

PCA $=$ Principal Component Analysis

$\mathrm{PCR}=$ Principal Component Regression

PLSR = Partial Least Squares Regression 


\section{List of Tables}

Table 1 the relationship between input and output valuables (correlation work)

Table 2 Distribution of the input and output variables

Table 3 An investigation on the number of input variables and neurons using TANSIG (transfer), TRAINBR (training) and LEARNGDM (learning) functions for 2 inputs (ANN model)

Table 4 An investigation on the number of input variables and neurons using TANSIG (transfer), TRAINBR (training) and LEARNGDM (learning) functions for 4 inputs (ANN model)

Table 5 Root Mean Square Error and correlation coefficient of prediction values for different MVR models used with four variables

Table 6 Values of different performance criteria for the models 


\section{List of Figures}

Figure 1 ANNs methodology flow chart

Figure 2 Distribution of the four input variables

Figure 3 The one hidden layer architecture for, [a] ANN2, four neurons and [b] ANN4 model, five neurons

Figure 4 Flow diagram of prediction steps of the NLR model

Figure 5 Predicted results of permeability using Simple Nonlinear Regression (NLR) model: (a) NLR2, using 2 inputs and (b) NLR4, using 4 inputs

Figure 6 Predicted results of permeability using Artificial Neural network (ANN) model: (a) ANN2, using 2 inputs and (b) ANN4, using 4 inputs

Figure 7 Measured permeability values vs. ANN4 with the two analytical models of permeability ( $\mathrm{K}-\mathrm{C}$ is Kozeny-Carman and H-B is Happel and Brenner) 
Table 1 the relationship between input and output valuables (correlation work)

\begin{tabular}{|l|l|c|}
\hline \multicolumn{1}{|c|}{ Input Variables } & $\begin{array}{c}\text { Correlation } \\
\text { with Output }\end{array}$ \\
\hline$X_{1}$ & Particle Size, $x_{S v}$ & 0.92 \\
\hline$X_{2}$ & Particle Concentration (v/v) & 0.20 \\
\hline$X_{3}$ & Shape Coefficient, $F_{v a}$ & 0.11 \\
\hline$X_{4}$ & $\boldsymbol{x}_{\mathbf{5 0}} / \boldsymbol{x}_{\mathbf{1 0}}$ & 0.06 \\
\hline
\end{tabular}

- Average particle size (Sauter mean diameter, $x_{s v}$ ),

- Solid concentration, $(C)$ by volume

- Particle shape coefficient $\left(F_{v a}\right)$ and

- Fines ratio $\left(x_{50} / x_{10}\right)$ 
Table 2 Distribution of the input and output variables

\begin{tabular}{|c|l|c|c|c|}
\hline & \multicolumn{1}{|c|}{ Variable } & Min & Max & Unit \\
\hline$X_{1}$ & Particle Size $\left(x_{s v}\right)$ & $0.245 \mathrm{E}-6$ & $168.0 \mathrm{E}-6$ & $\mathrm{~m}$ \\
\hline$X_{2}$ & Particle Concentration, $C(v / v)$ & 0.015 & 0.628 & - \\
\hline$X_{3}$ & Shape Coff. $\left(F_{v a}\right)$ & 0.0055 & 0.700 & - \\
\hline$X_{4}$ & $x_{50} / x_{10}$ & 1.150 & 29.410 & - \\
\hline$k$ & Measured Permeability & $1.63 \mathrm{E}-17$ & $5.3 \mathrm{E}-9$ & $\mathrm{~m}^{2}$ \\
\hline$k^{*}$ & Output & -0.788 & 7.724 & - \\
\hline
\end{tabular}


Table 3 An investigation on the number of input variables and neurons using TANSIG (transfer), TRAINBR (training) and LEARNGDM (learning) functions for 2 inputs (ANN model)

(a) Using Size and Concentration of particles

\begin{tabular}{|c|c|c|c|c|c|c|}
\hline$X_{1}$ & $X_{2}$ & $X_{3}$ & $X_{4}$ & Neuron & $\mathbf{R}^{2}$ & RMSE- \\
\hline$X_{S V}$ & $C$ & - & - & 1 & 0.901 & 0.167 \\
\hline$x_{S V}$ & $C$ & - & - & 2 & 0.947 & 0.123 \\
\hline$x_{S V}$ & $C$ & - & - & 3 & 0.962 & 0.105 \\
\hline$x_{S V}$ & $C$ & - & - & 4 & 0.980 & 0.075 \\
\hline$X_{S V}$ & $C$ & - & - & 6 & 0.945 & 0.127 \\
\hline$x_{S V}$ & $C$ & - & - & 10 & 0.940 & 0.129 \\
\hline$X_{S V}$ & $C$ & - & - & {$\left[\begin{array}{ll}1 & 1\end{array}\right]$} & 0.978 & 0.081 \\
\hline$x_{S V}$ & $C$ & - & - & {$\left[\begin{array}{ll}2 & 2\end{array}\right]$} & 0.979 & 0.079 \\
\hline$x_{S V}$ & C & - & - & {$\left[\begin{array}{ll}4 & 2\end{array}\right]$} & 0.980 & 0.074 \\
\hline
\end{tabular}

(b) Using Size with Shape Coefficient and with Size Distribution of particles

\begin{tabular}{|c|c|c|c|c|c|c|}
\hline$X_{1}$ & $X_{2}$ & $X_{3}$ & $X_{4}$ & Neuron & R $^{2}$ & RMSE- \\
\hline$X_{S V}$ & - & $F_{v a}$ & - & 4 & 0.949 & 0.121 \\
\hline$X_{S V}$ & - & - & $x_{50} / x_{10}$ & 4 & 0.913 & 0.157 \\
\hline
\end{tabular}

$X_{1}, X_{2}, X_{3}$ and $X_{4}$ : the input variables, RMSE: root mean square error, $x_{s v}$ : particle size (Sauter mean diameter), microns, $C$ : particle concentration $(v / v), x_{50} / x_{10}$ : particle size distribution, $F_{v a}$ : particle shape coefficient 
Table 4 An investigation on the number of input variables and neurons using TANSIG (transfer), TRAINBR (training) and LEARNGDM (learning) functions for 4 inputs (ANN model)

\begin{tabular}{|c|c|c|c|c|c|c|}
\hline$X_{1}$ & $X_{2}$ & $X_{3}$ & $X_{4}$ & Neuron & $\mathbf{R}^{2}$ & RMSE- \\
\hline$X_{S V}$ & $C$ & $F_{v a}$ & $x_{50} / x_{10}$ & 1 & 0.923 & 0.148 \\
\hline$x_{S V}$ & $C$ & $F_{v a}$ & $x_{50} / x_{10}$ & 3 & 0.971 & 0.093 \\
\hline$x_{S V}$ & $C$ & $F_{v a}$ & $x_{50} / x_{10}$ & 4 & 0.980 & 0.076 \\
\hline$x_{S V}$ & $C$ & $F_{v a}$ & $x_{50} / x_{10}$ & 5 & 0.990 & 0.054 \\
\hline$x_{S V}$ & $C$ & $F_{v a}$ & $x_{50} / x_{10}$ & 7 & 0.982 & 0.070 \\
\hline$x_{S V}$ & $C$ & $F_{v a}$ & $x_{50} / x_{10}$ & 11 & 0.970 & 0.094 \\
\hline$x_{S V}$ & $C$ & $F_{v a}$ & $x_{50} / x_{10}$ & {$\left[\begin{array}{ll}2 & 2\end{array}\right]$} & 0.903 & 0.167 \\
\hline$X_{S V}$ & $C$ & $F_{v a}$ & $x_{50} / x_{10}$ & {$\left[\begin{array}{ll}4 & 2\end{array}\right]$} & 0.955 & 0.116 \\
\hline$X_{S V}$ & $C$ & $F_{v a}$ & $x_{50} / x_{10}$ & {$\left[\begin{array}{ll}4 & 4\end{array}\right]$} & 0.968 & 0.097 \\
\hline
\end{tabular}

$X_{1}, X_{2}, X_{3}$ and $X_{4}$ : the input variables, RMSE: root mean square error, $x_{s v}$ : particle size (Sauter mean diameter), microns, $C$ : particle concentration $(v / v), x_{50} / x_{10}$ : particle size distribution, $F_{v a}$ : particle shape coefficient 
Table 5 Root Mean Square Error and correlation coefficient of prediction values for different MVR models used with four variables

\begin{tabular}{|c|c|c|c|}
\hline & PCR & PLSR & NLR \\
\hline RMSE & 0.553 & 0.556 & 0.0771 \\
\hline $\mathbf{R}^{\mathbf{2}}$ & 0.496 & 0.490 & 0.962 \\
\hline
\end{tabular}


Table 6 Values of different performance criteria for the models

\begin{tabular}{|c|c|c|c|c|}
\hline Model & Type of model & $\mathbf{R}^{2}$ & RMSE & AAE (\%) \\
\hline H-B & \multirow{2}{*}{ Analytical models } & - & - & 40.4 \\
\hline $\mathrm{K}-\mathrm{C}$ & & - & - & 35.2 \\
\hline NLR2 & \multirow{2}{*}{$\begin{array}{l}\text { Nonlinear models } \\
\text { with two inputs }\end{array}$} & 0.901 & 0.121 & 25.5 \\
\hline ANN2 & & 0.980 & 0.075 & 21.6 \\
\hline NLR4 & \multirow{2}{*}{$\begin{array}{l}\text { Nonlinear models } \\
\text { with four inputs }\end{array}$} & 0.962 & 0.077 & 14.3 \\
\hline ANN4 & & 0.990 & 0.054 & 9.6 \\
\hline
\end{tabular}




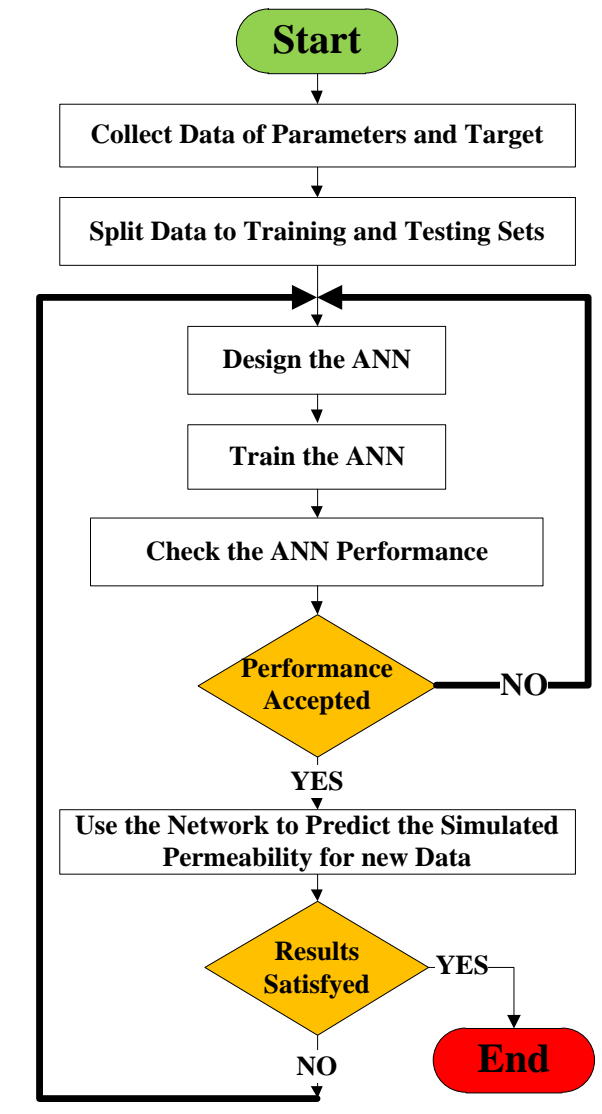

Figure 1 ANNs methodology flow chart 

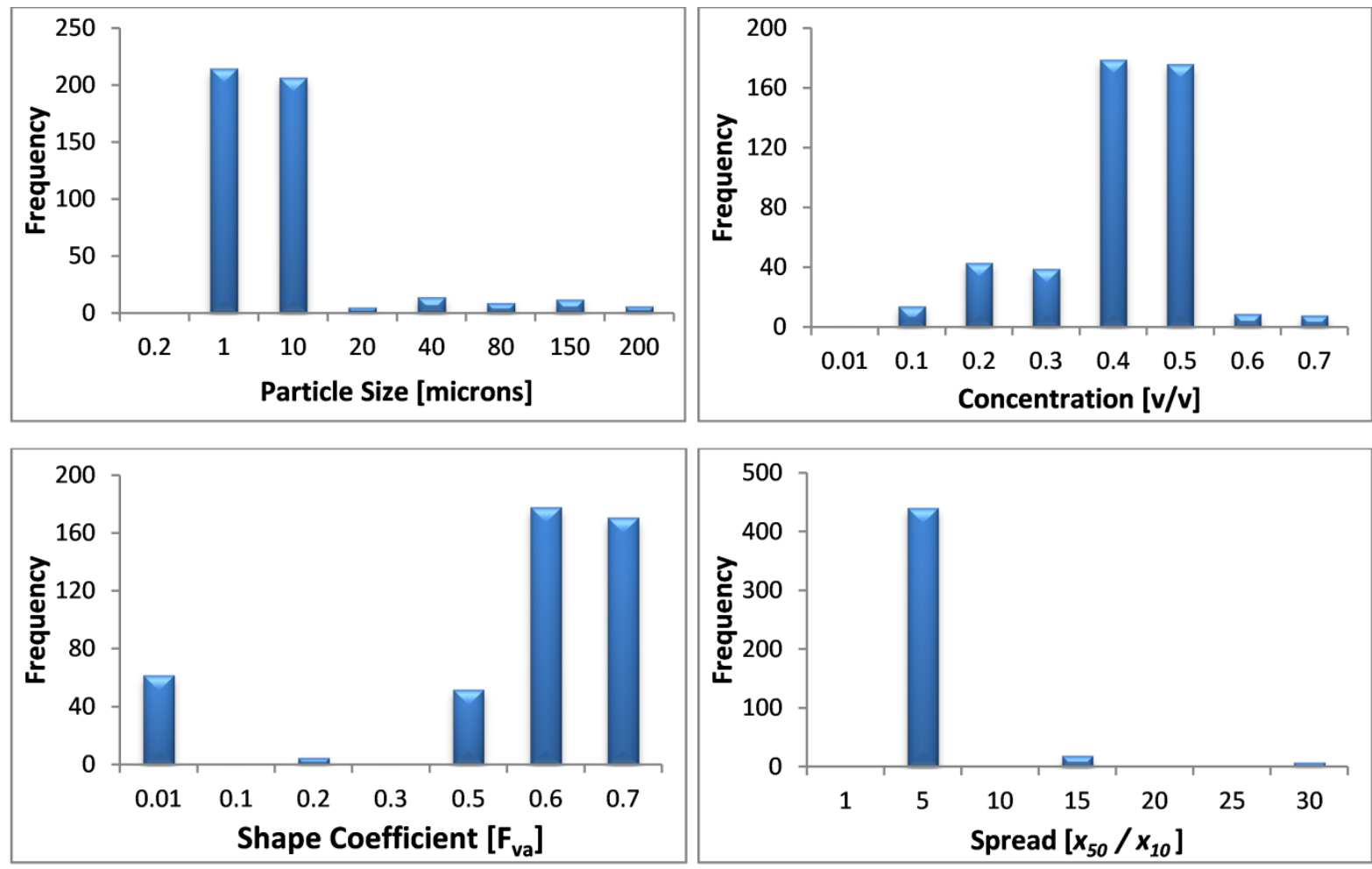

Figure 2 Distribution of the four input variables 

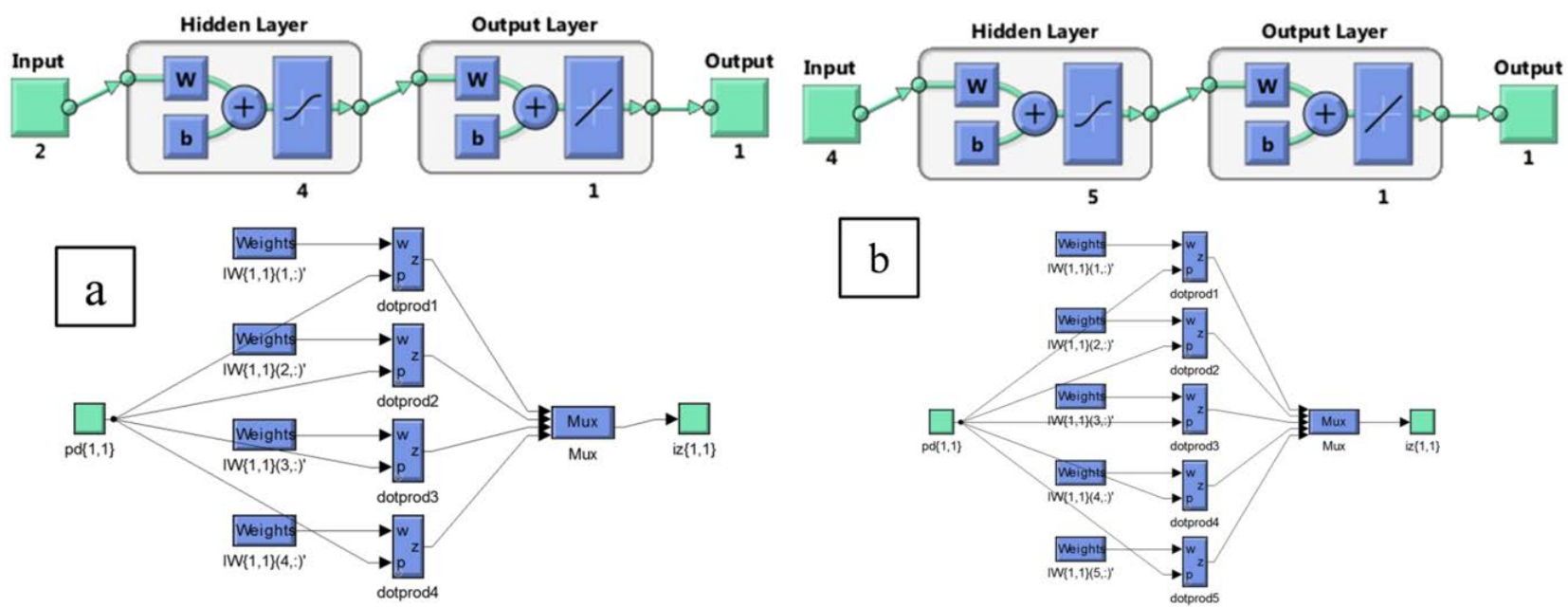

Figure 3 The one hidden layer architecture for, [a] ANN2, four neurons and [b] ANN4 model, five neurons 


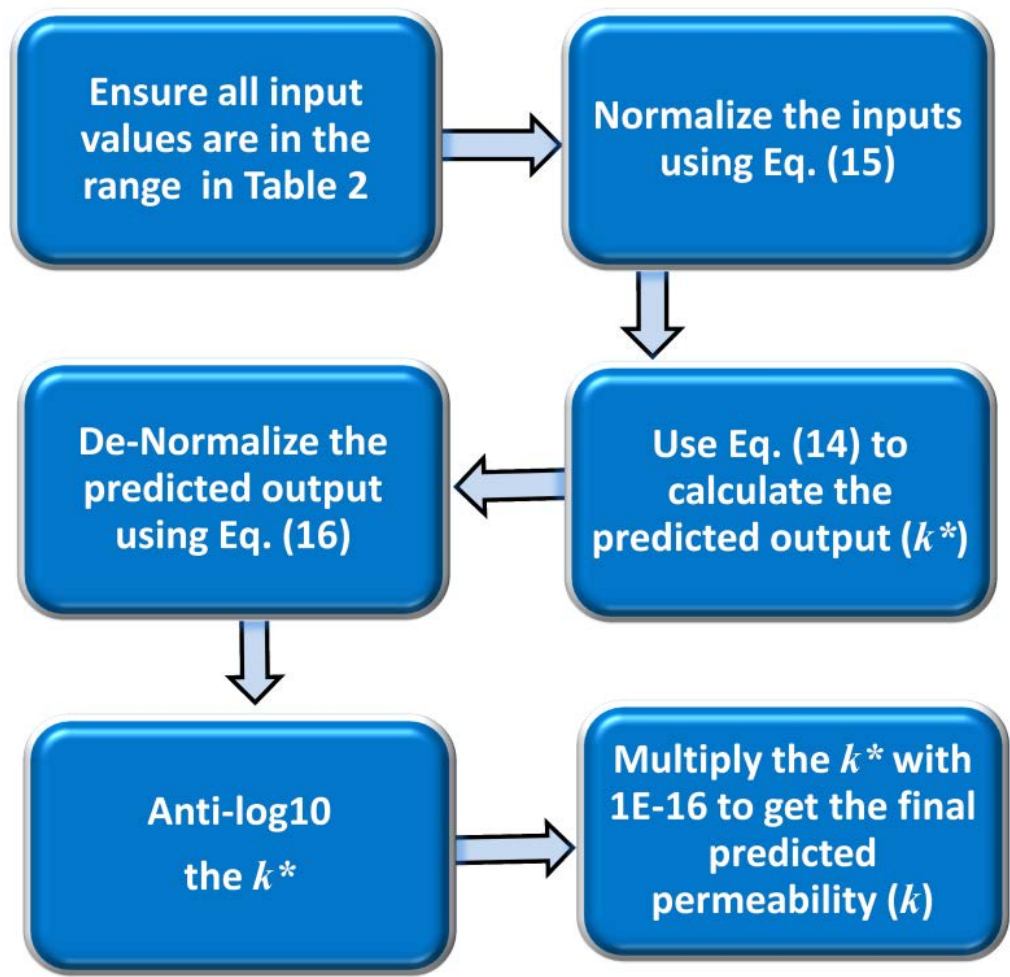

Figure 4 Flow diagram of prediction steps of the NLR model 

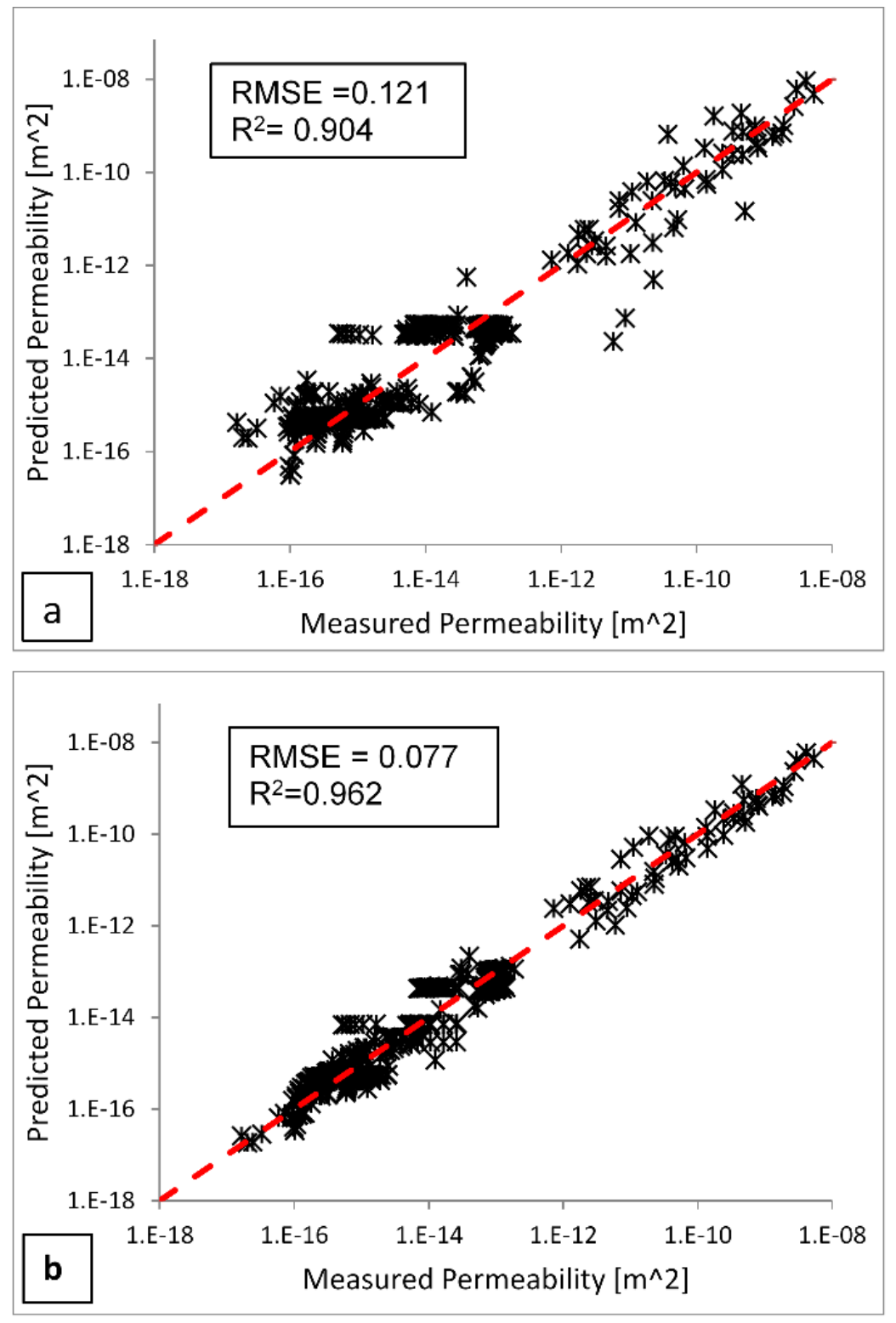

Figure 5 Predicted results of permeability using Simple Nonlinear Regression (NLR) model: (a) NLR2, using 2 inputs and (b) NLR4, using 4 inputs 

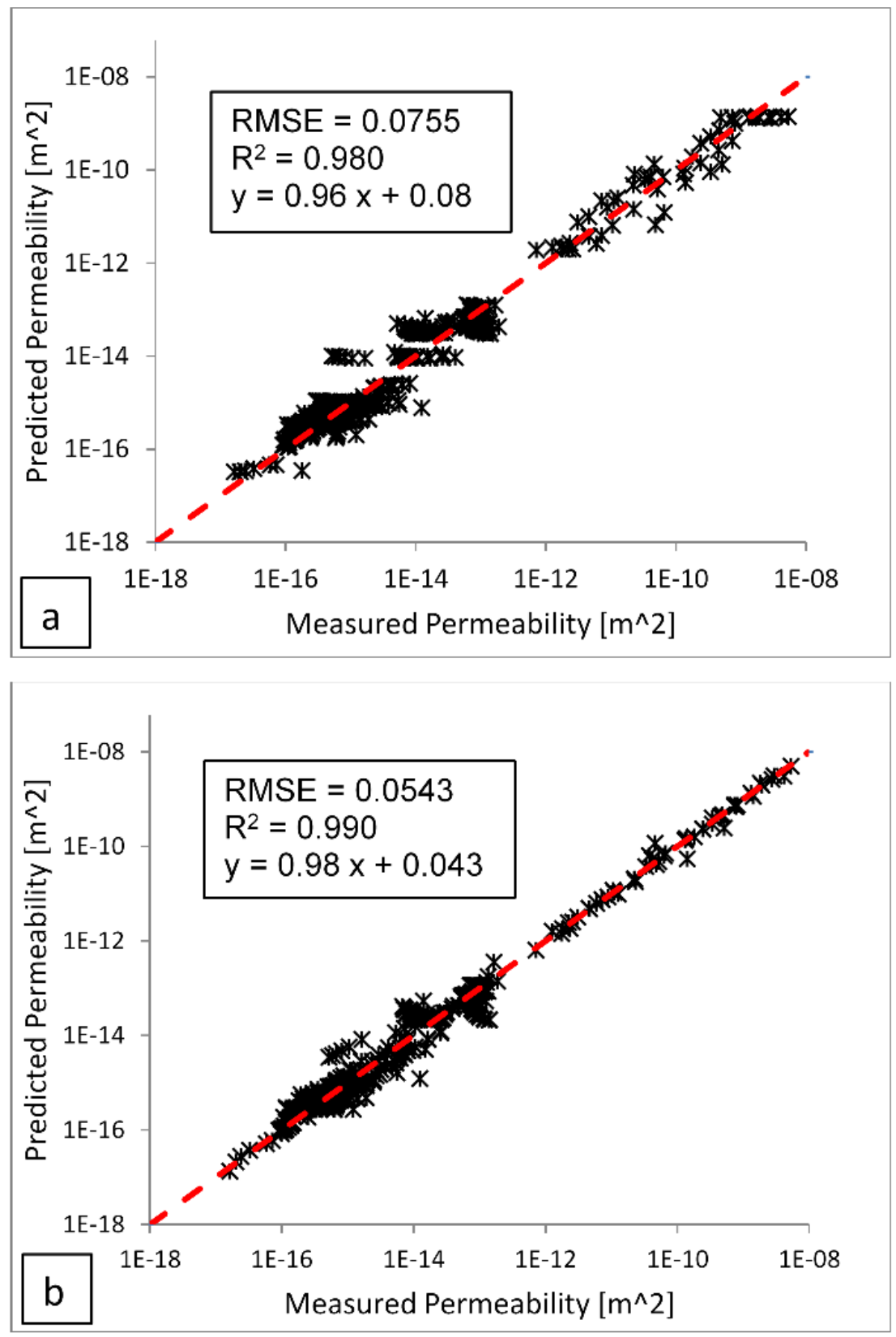

Figure 6 Predicted results of permeability using Artificial Neural network (ANN) model: (a) ANN2, using 2 inputs and (b) ANN4, using 4 inputs 


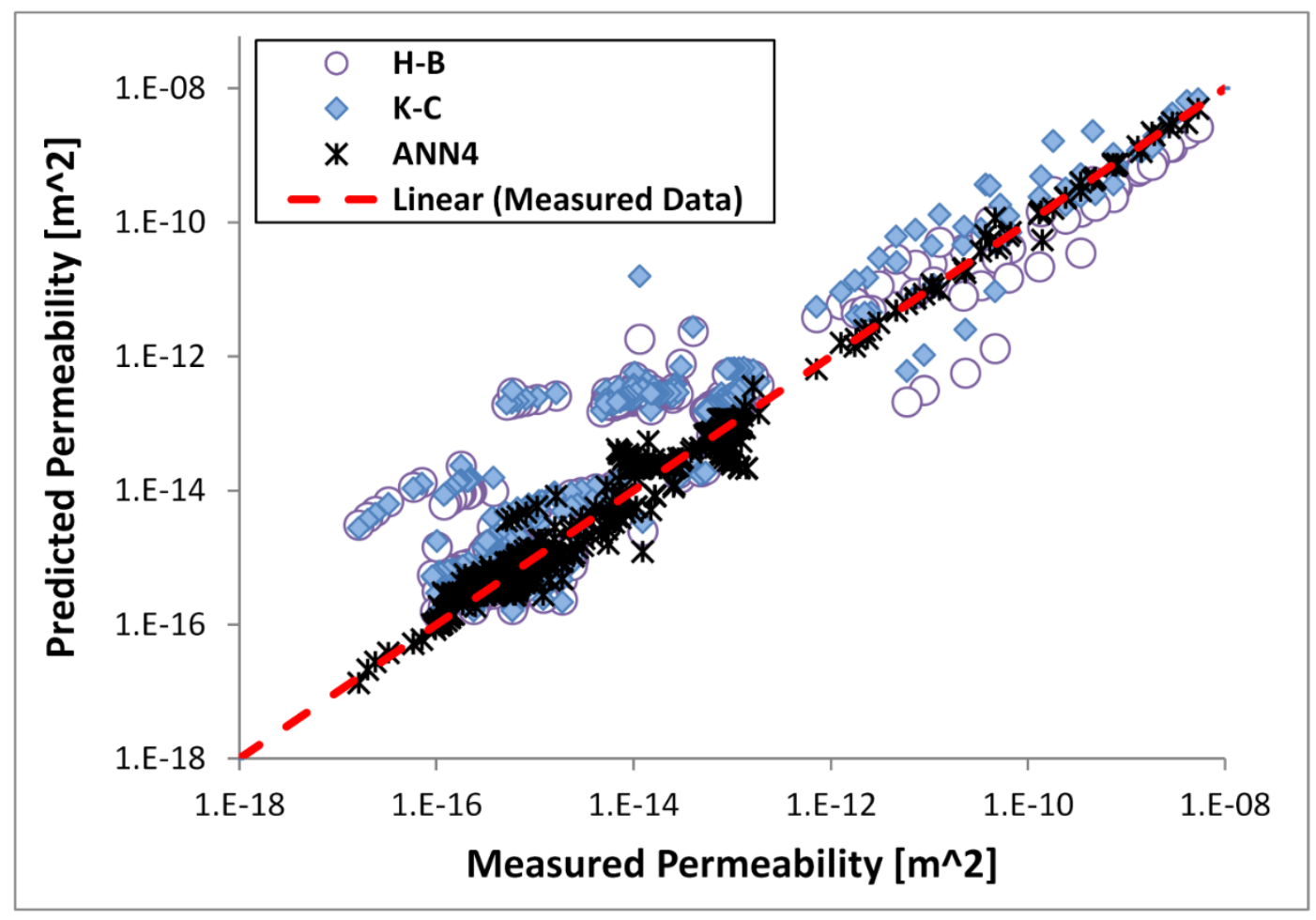

Figure 7 Measured permeability values vs. ANN4 with the two analytical models of permeability (K-C is Kozeny-Carman and $\mathrm{H}-\mathrm{B}$ is Happel and Brenner) 


\section{Appendix}

\section{Example of permeability prediction:}

Using an Excel spreadsheet and following the steps discussed in the flow diagram (Figure 4) it can be found that:

Step (1):

- $X_{1}=$ Sauter Mean Diameter : $\quad 4.827 \mathrm{E}-6 \mathrm{~m}$

- $X_{2}=$ Cake Concentration : $0.383 \mathrm{v} / \mathrm{v}$

- $X_{3}=$ Shape Coeff.: 0.700

- $X_{4}=$ Fines ratio $\left(x_{50} / x_{10}\right)$ : 2.630

Step (2): Normalize all values (inputs and the new output) using Eq. (8)

- $X_{1}=-0.945$

- $X_{2}=0.202$

- $X_{3}=1.000$

- $X_{4}=-0.895$

Step (3): Use Eq. (7) to predict the new output $\left(k^{*}=-0.102\right)$

Step (4): De-normalize the predicted output $\left(k^{*}\right)$ using Eq. (9) $\left(k^{\prime}=3.032\right)$

Step (5): Then anti- $\log 10(3.032)=1.076 \mathrm{E}+03$

Step (6): Finally, $k=1.076 \mathrm{E}+03 \times 1 \mathrm{E}-16=1.076 \mathrm{E}-13 \mathrm{~m}^{2}$, which is the final predicted permeability for these input conditions.

For comparison, the measured permeability for this material was $1.052 \mathrm{E}-13 \mathrm{~m}^{2}$, so the difference between the measured and the predicted values is small $\sim 2 \%$ using Eq. (6). 